\title{
Low Doses of the Anti-psychotic Drug Aripiprazole Have Strong P-gp-inhibitory Activity and Sensitize Anti-mitotic Drug-resistant Cancer Cells
}

\author{
JI YEONG KIM*, IN HWAN TAE*, BYUNG-MU LEE, HYUNG SIK KIM and SUNGPIL YOON \\ School of Pharmacy, Sungkyunkwan University, Suwon, Republic of Korea
}

\begin{abstract}
Background/Aim: The present study was designed to identify conditions that would increase the sensitivity of resistant cancer cells to anti-mitotic drugs. Materials and Methods: Previously, we showed that KBV20C cancer cells highly resistant to Halaven ${ }^{\circledR}$ (HAL) were sensitized by cotreatment with fluphenazine (FLU). In this study, we found that low doses of aripiprazole (ARI), another antipsychotic drug, sensitized HAL-resistant KBV20C cancer cells. We then investigated the mechanisms and roles of ARI in the sensitization of HAL-treated KBV20C cancer cells. Results: First-generation P-glycoprotein $(P$-gp) inhibitor verapamil required a dose that was nearly four-fold higher than that of ARI for P-gp inhibition, which suggested that ARI had a high specificity for $P$-gp binding to prevent efflux of anti-mitotic drugs. ARI was also found to sensitize HAL-treated KBV20C cells at a low dose, approximately 4-fold lower than that of verapamil. Co-treatment of ARI with another anti-mitotic drug, vincristine, also increased the sensitization of KBV20C cells. ARI caused a reduction in cell viability, increased $G_{2}$ arrest, and up-regulated expression of the DNA damage protein, pH2AX, when co-treated with HAL. Moreover, $G_{2}$ phase arrest and apoptosis in HAL-ARI cotreated cells resulted from the up-regulation of retinoblastoma protein, reduced extracellular signalregulated kinase pathway activity, and down-regulation of cell division cyclin protein. Conclusion: Cancer cells that are highly resistant to $H A L$ can be sensitized with the antipsychotic drug, ARI, which exerts specific P-gp inhibitory effects at a low dose.
\end{abstract}

\footnotetext{
*These Authors contributed equally to this work.

Correspondence to: Sungpil Yoon, Ph.D., School of Pharmacy, Sungkyunkwan University, 2066 Seobu-ro, Jangan-gu, Suwon, Gyeonggi-do, 16419, Republic of Korea. Tel: +82 1055024893, Fax: +82 312928800, e-mail: syoon88@gmail.com
}

Key Words: Aripiprazole, anti-psychotic drug, cancer, P-gp, drug resistance.
Anti-mitotic drugs, which target different binding sites on tubulin, are widely used for the treatment of different types of cancer $(1,2)$. These compounds inhibit mitosis by targeting microtubules and preventing their polymerization or depolymerization $(3,4)$. Eribulin, also called Halaven ${ }^{\circledR}$ (HAL), was recently developed to overcome the resistance of cancer cells to routinely used ant-mitotic drugs and used clinically for the treatment of resistant or metastatic cancer. HAL targets the depolymerization of microtubules $(5,6)$, and has shown promise for the treatment of triple-negative breast cancer and certain resistant cancer types (7-9). As patients are ultimately expected to develop resistance to HAL, identification of the mechanism(s) that underpin cell sensitization is an important step in the development of more effective treatments, and enables the design of approaches to increase HAL-associated apoptosis.

The overexpression of P-glycoprotein (P-gp) is a wellknown mechanism of resistance to anti-mitotic drugs in tumor cells (10). P-gp is a component of the cell membrane that is involved in the efflux of drugs, including anti-mitotics; its overexpression is one of the major mechanisms involved in multidrug resistance $(10,11)$. Despite the development of Pgp inhibitors, the toxicity of these agents to normal cells has resulted in the failure of clinical trials (10-13). Although the toxicity of first-generation P-gp inhibitors has prevented their clinical use, there have been several attempts to improve safety for further clinical trials $(11,12)$.

The purpose of the present study was to identify and investigate the efficacy of novel P-gp inhibitors with low toxicity in HAL-resistant cancer cells. The antipsychotic drug fluphenazine (FLU) was shown to exhibit inhibitory effects against P-gp and to have the ability to sensitize HALresistant cancer cells to treatment (14). In this study, we investigated the effect of aripiprazole (ARI), another antipsychotic drug, on the sensitization of HAL-resistant KBV20C cancer cells, and the mechanisms involved.

\section{Materials and Methods}

Reagents and cell culture. Rhodamine123, FLU, and verapamil (VER) were purchased from Sigma-Aldrich (St. Louis, MO, USA). Vincristine (VIC) was purchased from Enzo Life Sciences 
(Farmingdale, NY, USA). Aripiprazole (ARI) was purchased from Selleckchem (Houston, TX, USA). Aqueous solutions of HAL (Eisai Korea, Seoul, South Korea) were obtained from the National Cancer Center in South Korea.

Antibodies against p21 and cleaved poly ADP ribose polymerase (C-PARP) were obtained from Cell Signaling Technology (Danvers, MA, USA). Antibodies against $\beta$-actin, cyclin-dependent kinase 4 (CDK4), CDK2, phosphorylated-retinoblastoma protein $(\mathrm{pRb})$, cyclin D1, cyclin B1, cell division cyclin protein 2 (CDC2), phosphorylated-extracellular signal-regulated kinase (pERK), and cyclin E were obtained from Santa Cruz Biotechnology (Santa Cruz, CA, USA). Antibody against phosphorylated-H2A histone family member $\mathrm{X}$ (pH2AX) was obtained from Abcam (Cambridge, UK).

Human oral squamous carcinoma cell line KB and its multidrugresistant subline KBV20C were obtained from Dr. Yong Kee Kim (College of Pharmacy, Sookmyung Women's University, Seoul, Republic of Korea) and have been previously described (14-17). All cell lines were cultured in RPMI 1640 containing $10 \%$ fetal bovine serum, $100 \mathrm{U} / \mathrm{ml}$ penicillin, and $100 \mu \mathrm{g} / \mathrm{ml}$ streptomycin (WelGENE, Daegu, South Korea).

Microscopic observation. Cells were grown to $30-40 \%$ confluence in $60-\mathrm{mm}$ diameter dishes and treated for $24 \mathrm{~h}$ or $48 \mathrm{~h}$ with HAL, FLU, verapamil, or ARI, alone and in combination, or with $0.1 \%$ dimethylsulfoxide (DMSO; Control). The medium was removed, and phosphate-buffered saline (PBS) was added to each dish. Cells were examined immediately in two independent experiments using an ECLIPSE Ts2 inverted microscope (Nikon, Tokyo, Japan) with a $4 \times$ or a $10 \times$ objective lens.

Rhodamine uptake tests. The tests used to assess the ability of a drug to inhibit P-gp were based on a previously described method (14-17). Briefly, cells were grown to $30-40 \%$ confluence in $60-\mathrm{mm}$ diameter dishes and treated for $4 \mathrm{~h}$ or $24 \mathrm{~h}$ with verapamil, FLU, ARI, alone and in combination, or $0.1 \%$ DMSO. Cells were then incubated with $2 \mu \mathrm{g} / \mathrm{mI}$ rhodamine for $1 \mathrm{~h} 30 \mathrm{~min}$ at $37^{\circ} \mathrm{C}$. The medium was removed, and the cells were washed with PBS. The stained cells were analyzed for P-gp inhibition in two independent experiments using a Guava EasyCyte Plus Flow Cytometer (Merck Millipore, Billerica, MA, USA).

Fluorescence-activated cell sorting (FACS) analysis. FACS analysis was performed as previously described (18-21). Cells were grown to $30-40 \%$ confluence in $60-\mathrm{mm}$ diameter dishes and treated for 24 $\mathrm{h}$ with HAL, or ARI, alone and in combination, or with $0.1 \%$ DMSO. The cells were then dislodged by trypsin and pelleted by centrifugation. The pelleted cells were washed thoroughly with PBS, suspended in $75 \%$ ethanol for at least $1 \mathrm{~h}$ at $4^{\circ} \mathrm{C}$, washed with $\mathrm{PBS}$, and re-suspended in a cold propidium iodide (PI) staining solution $(100 \mu \mathrm{g} / \mathrm{ml}$ RNase A and $50 \mu \mathrm{g} / \mathrm{ml}$ PI in PBS) for $30 \mathrm{~min}$ at $37^{\circ} \mathrm{C}$. The stained cells were analyzed for cell-cycle distribution in two independent experiments for relative DNA content using a Guava EasyCyte Plus Flow Cytometer (Merck Millipore, Billerica, MA, USA).

Annexin $V$ analysis. Annexin $\mathrm{V}$ analysis was conducted using annexin V-fluorescein isothiocyanate (FITC) staining kit (BD Bioscience, Franklin, NJ, USA) as previously described (18-21). Cells were grown to $30-40 \%$ confluence in $60-\mathrm{mm}$ diameter dishes and treated for $24 \mathrm{~h}$ with HAL, verapamil, or ARI, alone and in combination, or with $0.1 \%$ DMSO. The cells were then dislodged by trypsin and pelleted by centrifugation. The pelleted cells were washed with PBS. Cells in $100 \mu \mathrm{I}$ of binding buffer received $5 \mu \mathrm{I}$ of Annexin V-FITC and $5 \mu \mathrm{I}$ of PI and were then incubated for 15 min at room temperature. The stained cells were analyzed for apoptosis in two independent experiments using a Guava EasyCyte Plus Flow Cytometer (Merck Millipore, Burlington, MA, USA).

Western blot analysis. KBV20C cells were grown to 30-40\% on 60 mm-diameter dishes and treated with HAL, or ARI, alone and in combination, or with $0.1 \%$ DMSO (Con). Total cellular proteins were then extracted as previously described (18-21). Briefly, cells were washed twice with cold PBS and detached with scrapers. For total protein isolation, cells were suspended in PRO-PREP ${ }^{\mathrm{TM}}$ protein extract solution (iNtRON, Seongnam, South Korea) and placed on ice for $30 \mathrm{~min}$. The suspension was collected after centrifugation at $15,000 \times g$ for $5 \mathrm{~min}$ at $4^{\circ} \mathrm{C}$. Protein concentrations were measured by using a protein assay kit (Bio-Rad, Hercules, CA, USA) according to the manufacturer's instructions. The proteins were resolved by sodium dodecyl sulfate-polyacrylamide gel electrophoresis and subjected to western blot analysis of $\beta$-actin, CDK4, CDK2, pRB, cyclin D1, cyclin B1, cyclin E, and pH2AX as previously described $(15,16)$.

Cell viability assay. Cell proliferation was measured by a colorimetric assay using EZ-CyTox cell viability assay kit (Daeillab, Seoul, South Korea) according to the manufacturer's instructions. Briefly, cells grown in wells of 96-well plates were treated for $48 \mathrm{~h}$ with HAL, FLU, ARI, alone and in combination, or with $0.1 \%$ DMSO. They were then incubated with $10 \mu \mathrm{l}$ of EZ-CyTox solution for $1-2 \mathrm{~h}$ at $37^{\circ} \mathrm{C}$. Development of orange color with formazan (absorbance at $450 \mathrm{~nm}$ ) was determined immediately using the VERSA MAX Microplate Reader (Molecular Devices Corp., Sunnyvale, CA, USA). All experiments were performed at least in triplicate and repeated twice.

Statistical analysis. Data are presented as mean \pm standard deviation (S.D.). Statistical analysis was performed by using Student's $t$-test and one-way analysis of variance (ANOVA) followed by a multiplecomparison test. Results were considered statistically significant compared to those of the control when $p<0.05$.

\section{Results}

HAL-treated KBV2OC cells were found to be more sensitized by co-treatment with ARI than with FLU. The KBV20C cell line is a very useful model of highly HAL-resistant cancer cells. We previously showed that the concentration of HAL required for a response in resistant cells similar to that of the parental drug-sensitive KB cells was approximately 500-fold higher $(14,17)$.

The antipsychotic drug FLU was shown to sensitize HALtreated KBV20C cells (14). We tested whether other antipsychotic drugs could sensitize HAL-treated KBV20C cells and found that ARI sensitized KBV20C cells to HAL. We conducted a more detailed analysis of ARI to support further clinical applications in anti-mitotic drug-resistant cancer cells. 
A

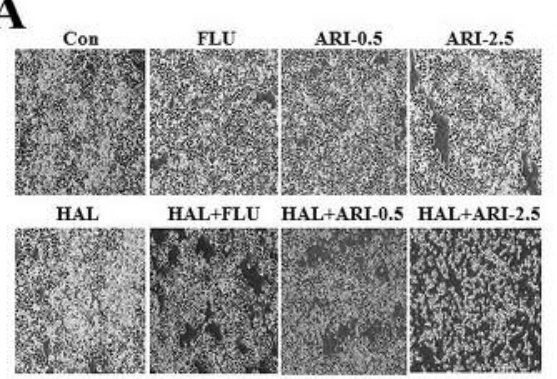

C
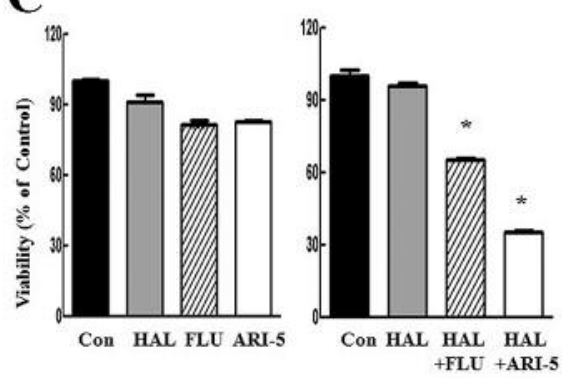

B

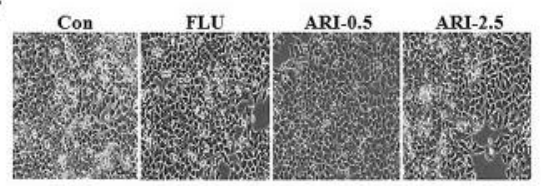

HAL

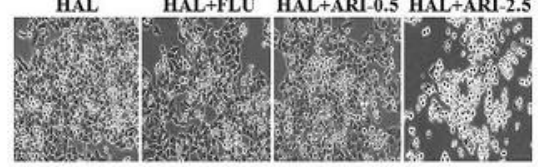

D

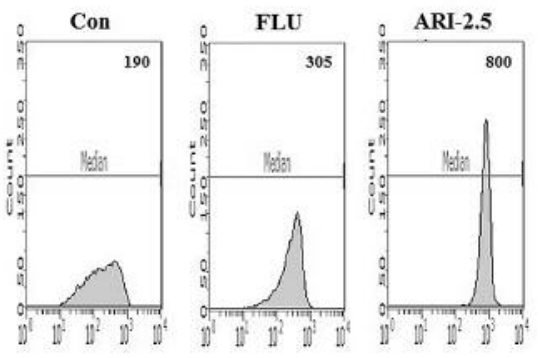

Figure 1. Co-treatment with the antipsychotic drug aripiprazole (ARI) increased sensitization of KBV2OC cells to Halaven (HAL) more than that caused by fluphenazine (FLU). A, B: KBV2OC cells were grown on $60 \mathrm{~mm}$-diameter dishes and treated with $50 \mathrm{ng} / \mathrm{ml} \mathrm{HAL}, 5 \mu \mathrm{M}$ FLU, or ARI at 0.5 $\mathrm{MM}$ (ARI-0.5) or $2.5 \mu M$ (ARI-2.5), alone and in combination, or with 0.1\% dimethylsulfoxide (DMSO; Con). After 1 day, cells were observed using an inverted microscope at $\times 4$ (A) or $\times 10$ (B) magnification (scale bar $=100 \mu \mathrm{m})$. C: KBV20C cells were plated on 96-well plates and grown to 30-40\%

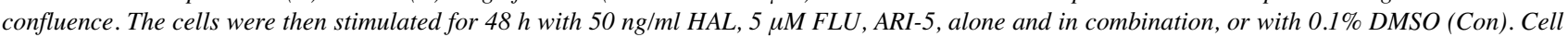
viability assay was then performed as described in the Materials and Methods. The data represent the mean \pm S.D. of at least two experiments repeated in triplicate. D: KBV20C cells were grown on $60 \mathrm{~mm}$-diameter dishes and treated with $10 \mu M$ FLU, ARI-2.5, or 0.1\% DMSO (Con). After 24 h, all cells were stained with rhodamine 123 and examined using Fluorescence-activated cell sorting analysis, as described in the Materials and Methods. *Significantly different at $p<0.05$ compared to the corresponding control (one-way analysis of variance followed by multiple-comparison test).

Firstly, we compared the effect of FLU and ARI on sensitivity of KBV20C cells to HAL. Microscopic observations revealed that ARI sensitized the cells to a similar degree as FLU at an approximately 10-fold lower concentration (Figure 1A and B). We confirmed these results using a viability assay (Figure $1 \mathrm{C}$ ). The results demonstrated that ARI was a more effective sensitizer of HAL-resistant cancer cells than FLU, which suggests that ARI might be used at a low dose or with lower drug toxicity to sensitize HAL-resistant cancer cells.

KBV20C cells exhibit a HAL-resistant phenotype via Pgp overexpression $(14,17)$. As FLU was demonstrated to sensitize KBV20C cells to HAL via P-gp inhibition (14), we also tested whether the increased sensitization by ARI was mediated through the inhibition of P-gp activity. Rhodamine 123, a well-known P-gp substrate, was used to measure Pgp inhibition $(16,17)$. In this experiment, the cellular accumulation of green fluorescence was indicative of the intracellular accumulation of rhodamine. As shown in Figure 1D, ARI more strongly inhibited P-gp activity than did FLU, which suggests that ARI binds to P-gp with higher specificity than FLU. We concluded that greater P-gp inhibitory action of ARI resulted in a stronger sensitization of KBV20C cells to HAL treatment.

Low doses of anti-psychotic drug ARI inhibited P-gp activity more strongly than VER. For further exploration of P-gp inhibition by ARI, we used comparison with the well-known first-generation P-gp inhibitor, $\operatorname{VER}(16,17)$. As shown in Figure 2A, ARI had a much stronger P-gp-inhibitory activity at much lower doses than did VER. This suggests that ARI had higher P-gp binding specificity than VER and might be used to minimize toxicity more effectively than VER. We also tested whether shorter ARI treatment times had a similar effect on P-gp inhibition. As shown in Figure 2B, treatment with ARI for $4 \mathrm{~h}$ led to similar results to those obtained with treatment with VER for $24 \mathrm{~h}$. This suggests that ARI inhibited P-gp via direct binding, similar to the inhibitory mechanism of VER. We also determined by how much the ARI dose could be reduced to achieve similar inhibitory activity to that of VER. As shown in Figure 2C, similar effects to VER were observed with four-fold lower dose of 


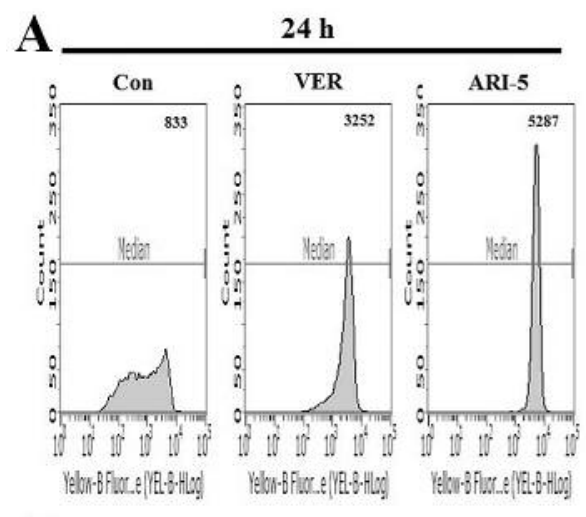

B
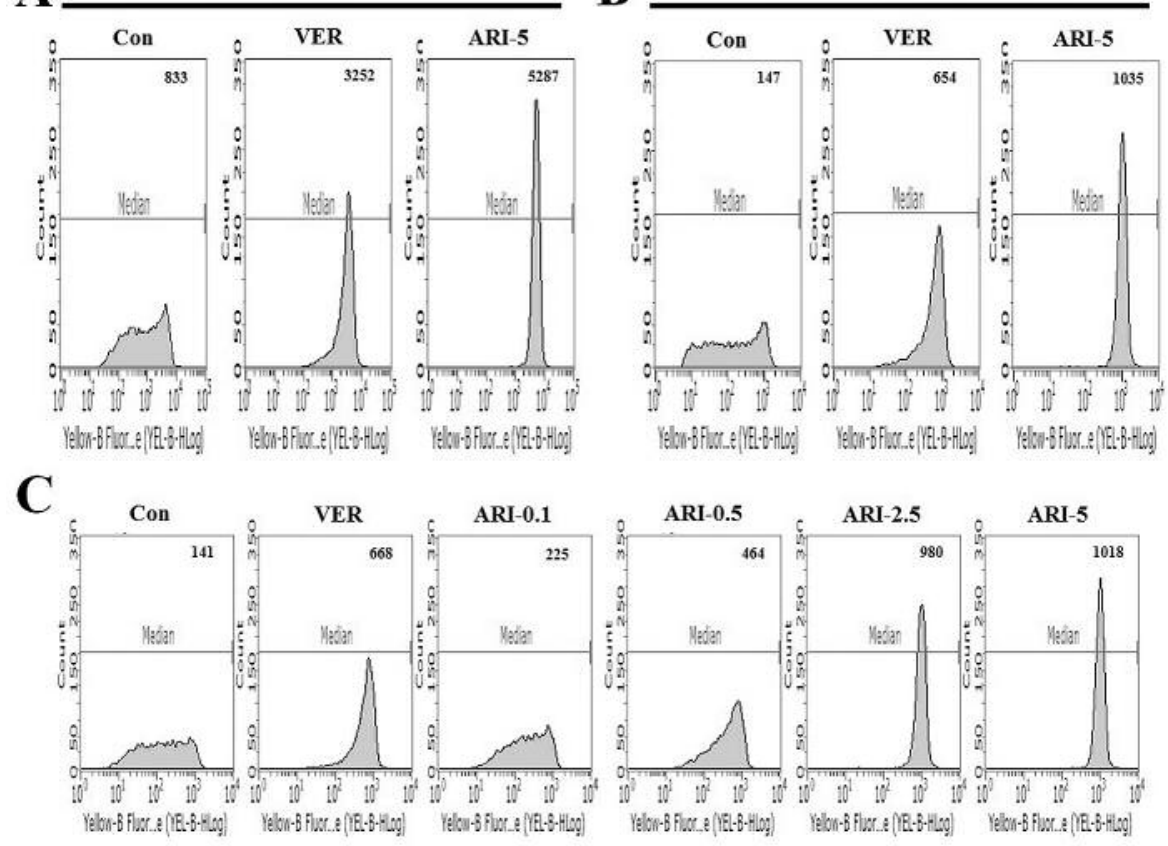

Figure 2. Low doses of the antipsychotic drug aripiprazole (ARI) inhibited P-gp more strongly than did verapamil (VER). KBV20C cells were grown on $60 \mathrm{~mm}$-diameter dishes and treated with $10 \mu \mathrm{M}$ verapamil (VER), or ARI at 0.1 $\mu M$ (ARI-0.1), $0.5 \mu M$ (ARI-0.5), $2.5 \mu M$ (ARI-2.5), or $5 \mu M$ (ARI-5), or 0.1\% dimethylsulfoxide (DMSO; Con) for $24 \mathrm{~h}$ (A and C) or $4 \mathrm{~h}(B)$. Cells were then stained with rhodamine 123 and examined using Fluorescence-activated cell sorting analysis as described in the Materials and Methods.

ARI. Both the shorter time and dose-dependent results of ARI treatment demonstrated the more specific P-gpinhibitory effects of ARI than those of VER. Hence, we propose that ARI can replace established P-gp inhibitors in clinical applications owing to its highly specific P-gp inhibition and lower toxicity.

Similar to the effects of VER, co-treatment with lower doses of ARI increased the sensitivity of KBV20C cells to HAL. We subsequently compared the sensitizing effect of VER and ARI on HAL treatment of KBV20C cells. As shown by microscopy (Figure 3A), $2.5 \mu \mathrm{M}$ ARI, and $10 \mu \mathrm{M}$ VER similarly sensitized the cells to HAL, reducing the number of viable KBV20C cells; this indicates that the lower dose of ARI was sufficient for sensitization of P-gp-overexpressing resistant cancer cells and more effective than VER. We also confirmed the results through the analysis of apoptosis using annexin $\mathrm{V}$ staining (Figure 3B). The results demonstrate that ARI was highly effective for the sensitization of HAL-resistant cancer cells. It also suggests that ARI can be used at lower doses or with reduced drug toxicity to sensitize HAL-resistant cancer.

Co-treatment with ARI increased apoptosis via DNA damage and ERK pathway in HAL-treated KBV20C cells. Furthermore, annexin $\mathrm{V}$ staining was used as a marker of apoptosis $(16,22)$. As shown in Figure 3B, co-treatment with HAL and ARI increased the number of cells in the early and late phases of apoptosis compared with ARI treatment alone, which suggests that apoptosis was the main cause of increased effect of the HAL-ARI co-treatment. When low $(0.5 \mu \mathrm{M})$ and high $(2.5 \mu \mathrm{M})$ doses of ARI were compared, dose-dependent increases were detected in the number of early and late apoptotic cells (Figure 3B). This suggests that increased P-gp inhibition caused by ARI led to the increased sensitization to HAL co-treatment. The induction of late apoptosis by HAL-ARI co-treatment occurred without the delay of early apoptosis (Figure 3B), which suggests that the HAL-ARI combination was highly effective for the sensitization of resistant cancer cells. To confirm that apoptosis was increased in co-treated cell, we measured C-PARP production as a molecular marker $(15,16)$. As shown in Figure 3C, C-PARP expression increased in HALARI co-treated cells. This indicates that co-treatment of ARI with HAL increased the apoptosis of drug-resistant KBV20C cells. Collectively, these data indicate that co-treatment with the antipsychotic drug ARI sensitized KBV20C cells to HAL treatment at a much higher efficacy than that of VER.

In the next phase of our investigation, we tested the expression of proteins involved in DNA damage and cellular signaling pathways. As shown in Figure 3C, we found that 

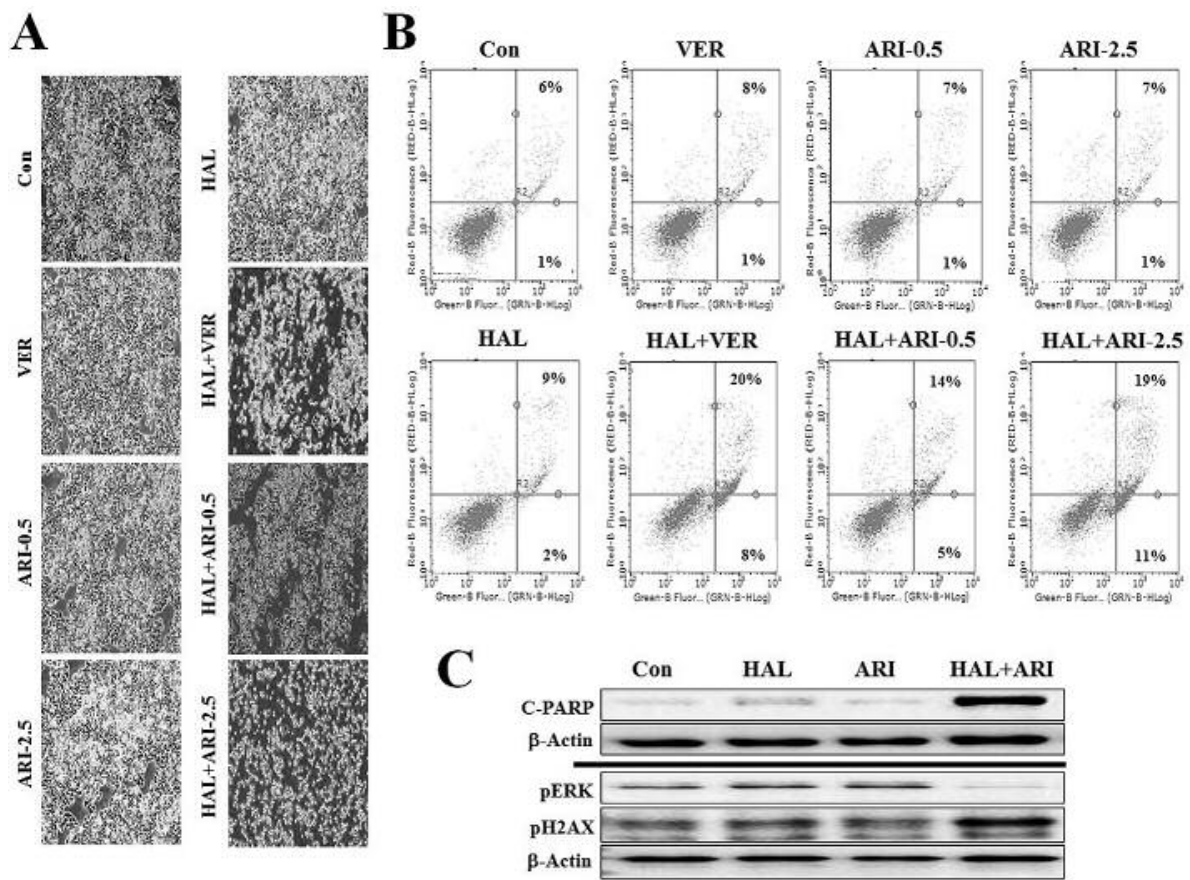

Figure 3. Co-treatment with aripiprazole (ARI) increased apoptosis via DNA damage and extracellular signal-regulated kinase (ERK) pathway down-regulation in Halaven (HAL)-treated KBV20C cells. KBV2OC cells were grown on $60 \mathrm{~mm}$-diameter dishes and treated with $50 \mathrm{ng} / \mathrm{ml} \mathrm{HAL}$, $10 \mu M$ verapamil (VER), or ARI at $0.5 \mu M$ (ARI-0.5), $2.5 \mu M$ (ARI-2.5), alone and in combination or with $0.1 \%$ DMSO (Con). After 1 day, cells were observed using an inverted microscope at $\times 4$ magnification $(A)$, and analyzed by annexin $V$ assay $(B)$, and by western blot using antibodies against cleaved poly ADP ribose polymerase (C-PARP), phosphorylated-extracellular signal-regulated kinase ( $p E R K)$, phosphorylated-H2A histone family member $X(\mathrm{pH} 2 \mathrm{AX})$, and $\beta$-actin $(\mathrm{C})$.

pH2AX, a protein marker of DNA damage, was largely increased following co-treatments. In analysis of growth signaling, we found that pERK was down-regulated by HAL-ARI co-treatment (Figure 3C). These results suggest that DNA damage and down-regulation of the pERK pathway may lead to increased apoptosis in HAL-ARI cotreated KBV20C cells.

Co-treatment of KBV20C cells with ARI and HAL induced $G_{2}$ arrest. To clarify the mechanism of action of the HALARI combination, FACS analyses were performed. As shown in Figure 4A, co-treatment with HAL and ARI led to more cells being found in $G_{2}$ arrest than those induced by individual treatment with either agent. A positive relationship was also detected between the dose of ARI and the proportion of HAL-treated cells in $\mathrm{G}_{2}$ arrest (Figure 4A), which suggests that increased P-gp inhibition prevented continuous efflux of HAL from inside KBV20C cells. This indicates that the increase in apoptosis was stimulated with cell-cycle arrest.

To investigate further the expression of proteins involved in $\mathrm{G}_{2}$ arrest $(15,16,20)$, we performed western blotting. As shown in Figure 4B, there were no significant differences in the expression of CDK2, CDK4, cyclin E, and p21 proteins. Although small increases were found in cyclin B1 and cyclin D1 after co-treatment, we assumed that the changes were negligible and did not contribute extensively to sensitization by HAL-ARI co-treatment. Importantly, pRB and CDC2 levels were significantly altered following co-treatments, which suggests that these cell cycle-related proteins may increase $\mathrm{G}_{2}$ arrest in HAL-ARI co-treated KBV20C cells. Collectively, the co-treatment of ARI with HAL increased apoptosis of drugresistant KBV20C cells via $\mathrm{G}_{2}$ cell-cycle arrest.

Low doses of ARI increased sensitization of VIC-treated KBV20C cells. We demonstrated the strong P-gp inhibition of ARI (Figure 2). We tested whether ARI inhibited the efflux of other anti-mitotic drugs. We tested VIC, which is an anti-mitotic drug routinely used in treatment of patients with cancer $(22,23)$. As shown in Figure $4 \mathrm{C}$, when combined with VIC, low doses of ARI had a similar sensitizing effect to that seen with HAL-co-treatment. This suggests that low doses of ARI might be combined with other anti-mitotic drugs to prevent their efflux. We conclude that ARI can be used for the treatment of patients with various types of $\mathrm{P}$-gp-overexpressing drug-resistant cancer. 
A
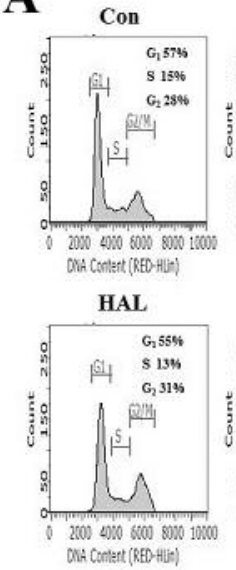

C

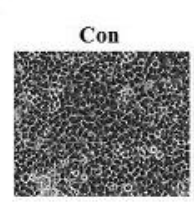

ARI-0.5

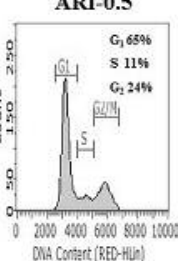

HAL+ARI-0.5

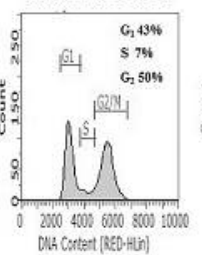

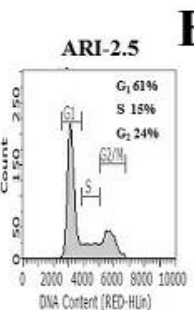
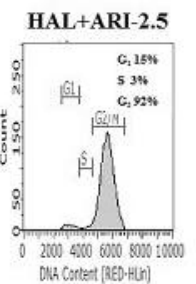

B Con HAL ARI HAL +ARI
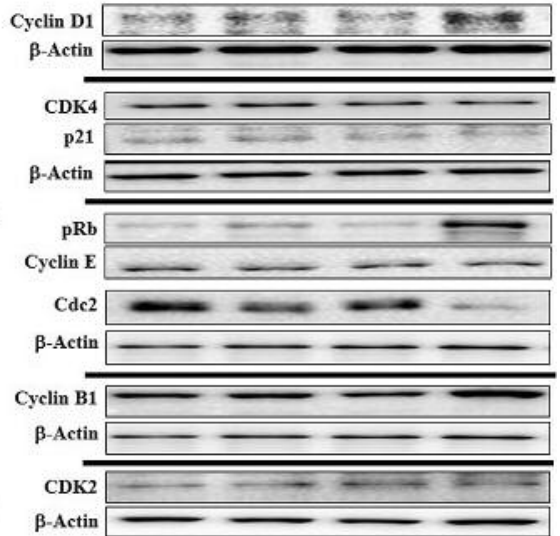

$\beta$-Actin

ARI-2.5

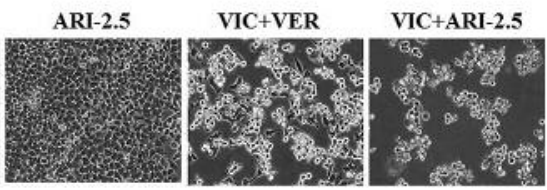

Figure 4. Co-treatment of KBV2OC cells with aripiprazole (ARI) and Halaven (HAL) induced $G_{2}$ cell-cycle arrest. Low doses of ARI also sensitized KBV20C cells to vincristine (VIC) treatment. KBV20C cells were grown on $60 \mathrm{~mm}$-diameter dishes and treated with $50 \mathrm{ng} / \mathrm{ml} \mathrm{HAL,} \mathrm{ARI} \mathrm{at} 0.5 \mu \mathrm{M}$ (ARI-0.5) or $2.5 \mu M$ (ARI-2.5), alone and in contestation, or with 0.1\% DMSO (Con). After $24 \mathrm{~h}$, Fluorescence-activated cell sorting analyses were performed as described in the Materials and Methods (A) and western blot analysis was performed using antibodies against cyclin D1, cyclin-dependent kinase 4 (CDK4), p21, phosphorylated-retinoblastoma protein (pRb), cell division cyclin protein 2 (CDC2), cyclin B1, cyclin E, CDK2, and $\beta$-actin (B). C: KBV20C cells were grown on $60 \mathrm{~mm}$-diameter dishes and treated with $5 \mathrm{nM}$ VIN, $10 \mu \mathrm{M}$ verapamil (VER-10), or ARI at 2.5 $\mu M$ (ARI-2.5), alone and in combination, or with $0.1 \%$ DMSO (Con). After 1 day, all cells were observed using an inverted microscope at $\times 10$ magnification.

\section{Discussion}

Previously, we found that P-gp-overexpressing KBV20C cells were highly resistant to $\operatorname{HAL}(14,17)$. Therefore, KBV20C cells are useful as models of highly HAL-resistant cancer. We assumed that our studies using KBV20C cancer cells might provide insight into the treatment of patients with HAL-resistant cancer. HAL was recently developed, and is especially promising for the treatment of patients in whom anticancer drugs have previously failed $(5,6,8)$. As it is expected that patients with cancer treated with HAL will ultimately develop resistance, our results suggest a novel method for the sensitization of patients with HAL-resistant cancer.

We reported the HAL-sensitizing effects of the antipsychotic drug FLU in KBV20C cells (14). The cancer cell-sensitizing ability of ARI has also been demonstrated in different cancer models (24-26), suggesting it to be a potential chemotherapeutic agent. In this study, the effects of HAL-ARI co-treatments were analyzed to our knowledge for the first time. We found that ARI has much stronger sensitizing effects than FLU in HAL-treated KBV20C cancer cells, which suggests that ARI can be used at lower doses in combination with HAL thereby reducing toxicity to normal cells. As the efflux of HAL by P-gp is the main mechanism for the resistance of KBV20C cells to HAL, we examined whether the sensitization resulted from the inhibitory activity of ARI. We demonstrated that ARI exerted strong P-gp inhibition to prevent the efflux of HAL. ARI was found to have approximately 10-fold stronger P-gp inhibitory activity than FLU, which suggests that the stronger sensitization effect of the HAL-ARI co-treatment resulted from strong P-gp inhibition by ARI. This also indicates that the main mechanism of the sensitization of the HAL-ARI treatment resulted from the highly specific P-gp inhibitory activity of ARI.

Furthermore, we compared P-gp inhibitory activity between ARI and the first-generation P-gp inhibitor VER $(10,13)$. An approximately four-fold lower dose of ARI than VER was required for similar inhibitory activity against KBV20C cells. These results suggest that ARI is a highly specific inhibitor of P-gp in HAL-resistant cancer cells. Our results also demonstrate it was possible to achieve P-gp inhibition by ARI treatment even with a short treatment time; this suggests that ARI inhibits $\mathrm{P}$-gp activity via direct binding to $\mathrm{P}$-gp for the subsequent prevention of $\mathrm{P}$-gpmediated drug efflux. As VER is a representative and 
established P-gp inhibitor $(10,16,17)$, ARI should be regarded as a very strong P-gp inhibitor. Moreover, as P-gp inhibitors are reportedly toxic to normal cells $(10,11,13)$, our findings may contribute to the development of ARI as a low-dose P-gp inhibitor, in combination with HAL, for sensitization of cancer cells. We believe that repositioned drugs such as ARI can easily be administered to patients with HAL-resistant cancer.

More importantly, our results were not limited by HAL co-treatment. We confirmed that ARI had a similar sensitizing effect with VIC in P-gp-overexpressing KBV20C cells. They also indicate that ARI sensitizes other antimitotic drug-resistant cancer cells via the inhibition of drug efflux. We believe that ARI can generally be used in combination with other cancer drugs for sensitizing resistant cancer cells. However, further in vivo studies using animal models are required to assess the efficacy of the combination therapy of anti-mitotic drugs and ARI before use in clinical trials.

We demonstrated that HAL-ARI co-treatment reduced cell proliferation and increased $\mathrm{G}_{2}$ arrest in highly HAL-resistant KBV20C cells. From microscopy, FACS analysis, annexin V staining, and western blotting, we concluded that apoptosis was increased by HAL-ARI co-treatment via increased $\mathrm{G}_{2}$ arrest and reduced proliferation. Furthermore, a marker of DNA damage, $\mathrm{pH} 2 \mathrm{AX}$, the cell-cycle protein $\mathrm{pRb}$, and the pro-apoptotic protein, C-PARP, were increased after HALARI co-treatment. This indicates that the mechanism of action involved $\mathrm{G}_{2}$ phase arrest via DNA damage and an increase in the number of apoptotic cells. We hypothesized that ARI prevented HAL efflux; subsequently, the retained HAL stimulated $G_{2}$ arrest through an increase in DNA damage and reduced cellular proliferation, thereby increasing apoptosis of HAL-treated resistant KBV20C cells. In addition, the combination of ARI and HAL reduced the expression of CDC2 and pERK, which indicates that these proteins may also be involved in the effects of the HAL-ARI co-treatment.

Collectively, our results showed that drug-resistant KBV20C cells overexpressing P-gp can be sensitized to antimitotic drug treatment (HAL or VIC) in combination with antipsychotic drug (FLU or ARI). Most importantly, we found that ARI was highly selective for the sensitization of anti-mitotic drug-resistant cancer cells via inhibition of P-gp activity. As these drugs are already used in clinical settings, drug reposition would offer an efficient method to address the urgent need for pharmacological treatment of drugresistant cancer, allowing the treatment of patients with drugresistant disease at a relatively fast pace.

\section{Conflicts of Interest}

The Authors declare no conflict of interest in regard to this study.

\section{Acknowledgements}

The Authors thank Yujin Park for help in technical support and preparation of the article. This research was supported by National Research Foundation of Korea (NRF) funded by the Ministry of Education (NRF-2017R1D1A1B03029158).

\section{References}

1 Jordan MA and Wilson L: Microtubules as a target for anticancer drugs. Nat Rev Cancer 4: 253-265, 2004.

2 McGrogan BT, Gilmartin B, Carney DN and McCann A: Taxanes, microtubules and chemoresistant breast cancer. Biochim Biophys Acta 1785: 96-132, 2008.

3 Matsuo K, Bond VK, Im DD and Rosenshein NB: Prediction of chemotherapy response with platinum and taxane in the advanced stage of ovarian and uterine carcinosarcoma: A clinical implication of in vitro drug resistance assay. Am J Clin Oncol 33: 358-363, 2010.

4 Yang $\mathrm{H}$ and Dou QP: Targeting apoptosis pathway with natural terpenoids: implications for treatment of breast and prostate cancer. Curr Drug Targets 11: 733-744, 2010.

5 Dell'Ova M, De Maio E, Guiu S, Roca L, Dalenc F, Durigova A, Pinguet F, Bekhtari K, Jacot W and Pouderoux S: Tumour biology, metastatic sites and taxanes sensitivity as determinants of eribulin mesylate efficacy in breast cancer: results from the ERIBEX retrospective, international, multicenter study. BMC Cancer 15: 659, 2015.

6 Laughney AM, Kim E, Sprachman MM, Miller MA, Kohler RH, Yang KS, Orth JD, Mitchison TJ and Weissleder R: Single-cell pharmacokinetic imaging reveals a therapeutic strategy to overcome drug resistance to the microtubule inhibitor eribulin. Sci Transl Med 6: 261ra152, 2014.

7 Aogi K, Iwata H, Masuda N, Mukai H, Yoshida M, Rai Y, Taguchi K, Sasaki Y and Takashima S: A phase II study of eribulin in Japanese patients with heavily pretreated metastatic breast cancer. Ann Oncol 23: 1441-1448, 2012.

8 Dybdal-Hargreaves NF, Risinger AL and Mooberry SL: Eribulin mesylate: mechanism of action of a unique microtubule-targeting agent. Clin Cancer Res 21: 2445-2452, 2015.

9 Inoue K, Saito T, Okubo K, Kimizuka K, Yamada H, Sakurai T, Ishizuna K, Hata S, Kai T and Kurosumi M: Phase II clinical study of eribulin monotherapy in Japanese patients with metastatic breast cancer who had well-defined taxane resistance. Breast Cancer Res Treat 157: 295-305, 2016.

10 Chen Z, Shi T, Zhang L, Zhu P, Deng M, Huang C, Hu T, Jiang $\mathrm{L}$ and Li J: Mammalian drug efflux transporters of the ATP binding cassette $(\mathrm{ABC})$ family in multidrug resistance: A review of the past decade. Cancer Lett 370: 153-164, 2016.

11 Chufan EE, Kapoor K and Ambudkar SV: Drug-protein hydrogen bonds govern the inhibition of the ATP hydrolysis of the multidrug transporter P-glycoprotein. Biochem Pharmacol 101: 40-53, 2016.

12 Shukla S, Wu CP and Ambudkar SV: Development of inhibitors of ATP-binding cassette drug transporters: Present status and challenges. Expert Opin Drug Metab Toxicol 4: 205-223, 2008.

13 Yang K, Wu J and Li X: Recent advances in the research of Pglycoprotein inhibitors. Biosci Trends 2: 137-146, 2008.

14 Cheon JH, Lee BM, Kim HS and Yoon S: Highly Halavenresistant KBV20C cancer cells can be sensitized by co-treatment with fluphenazine. Anticancer Res 36: 5867-5874, 2016. 
15 Cheon JH, Kim JY, Lee BM, Kim HS and Yoon S: P-gp Inhibition by XL019, a JAK2 inhibitor, increases apoptosis of vincristine-treated resistant KBV20C cells with increased p21 and pH2AX expression. Anticancer Res 37: 6761-6769, 2017.

16 Cheon JH, Kim KS, Yadav DK, Kim M, Kim HS and Yoon S: The JAK2 inhibitors CEP-33779 and NVP-BSK805 have high P-gp inhibitory activity and sensitize drug-resistant cancer cells to vincristine. Biochem Biophys Res Commun 490: 1176-1182, 2017.

17 Park Y, Son JY, Lee BM, Kim HS and Yoon S: Highly eribulinresistant KBV20C oral cancer cells can be sensitized by cotreatment with the third-generation P-glycoprotein inhibitor, elacridar, at a low dose. Anticancer Res 37: 4139-4146, 2017.

18 Choi AR, Kim JH, Cheon JH, Kim HS and Yoon S: Attenuation of colchicine toxicity in drug-resistant cancer cells by cotreatment with anti-malarial drugs. Anticancer Res 36: 58595866, 2016.

19 Choi AR, Kim JH, Woo YH, Cheon JH, Kim HS and Yoon S: Co-treatment of LY294002 or MK-2206 with AZD5363 attenuates AZD5363-induced increase in the level of phosphorylated AKT. Anticancer Res 36: 5849-5858, 2016.

20 Choi AR, Kim JH, Woo YH, Kim HS and Yoon S: Anti-malarial drugs primaquine and chloroquine have different sensitization effects with anti-mitotic drugs in resistant cancer cells. Anticancer Res 36: 1641-1648, 2016.

21 Lim JS, Park Y, Lee BM, Kim HS and Yoon S: Co-treatment with celecoxib or NS398 strongly sensitizes resistant cancer cells to anti-mitotic drugs independent of P-gp Inhibition. Anticancer Res 36: 5063-5070, 2016.
22 Wang LG, Liu XM, Kreis W and Budman DR: The effect of antimicrotubule agents on signal transduction pathways of apoptosis: A review. Cancer Chemother Pharmacol 44: 355-361, 1999.

23 Florian S and Mitchison TJ: Anti-microtubule drugs. Methods Mol Biol 1413: 403-421, 2016.

24 Johnston AN, Bu W, Hein S, Garcia S, Camacho L, Xue L, Qin L, Nagi C, Hilsenbeck SG, Kapali J, Podsypanina K, Nangia J and Li Y: Hyperprolactinemia-inducing antipsychotics increase breast cancer risk by activating JAK-STAT5 in precancerous lesions. Breast Cancer Res 20: 42, 2018.

25 Kim MS, Yoo BC, Yang WS, Han SY, Jeong D, Song JM, Kim $\mathrm{KH}$, Aravinthan A, Kim JH, Kim JH, Kim SC and Cho JY: SRC is the primary target of aripiprazole, an atypical antipsychotic drug, in its anti-tumor action. Oncotarget 9: 5979-5992, 2018.

26 Suzuki S, Okada M, Kuramoto K, Takeda H, Sakaki H, Watarai $\mathrm{H}$, Sanomachi $\mathrm{T}$, Seino S, Yoshioka $\mathrm{T}$ and Kitanaka C: Aripiprazole, an antipsychotic and partial dopamine agonist, inhibits cancer stem cells and reverses chemoresistance. Anticancer Res 36: 5153-5161, 2016.
Received July 27, 2018

Revised August 6, 2018

Accepted August 7, 2018 